8. Hoffmann GF, Charpentier C, Mayatepek E, Mancini J, Leichsenring $\mathrm{M}$, Gibson $\mathrm{KM}$, et al. Clinical and biochemical phenotype in 11 patients with mevalonic aciduria. Pediatrics. 1993;91:915-21.

9. Church LD, Churchman SM, Hawkins PN, McDermott MF. Hereditary auto-inflammatory disorders and biologics. Springer Semin Immunopathol. 2006;27: 494508.

10. Topaloðlu R, Ayaz NA, Waterham HR, Yüce A, Gumruk F, Sanal O. Hyperimmunoglobulinemia D and periodic fever syndrome; treatment with etanercept and follow-up. Clin Rheumatol. 2008;27:1317-20.

\title{
Mutation Analysis of Indian Patients with Urea Cycle Defects
}

\author{
NeERJa Gupta, Madhulika Kabra AND *J HäBERLE \\ From the Genetic Unit, Department of Pediatrics, AIIMS and *Kinderspital, Division of Metabolism, University Children’s Hospital \\ Steinwiesstr. 75, 8032 Zurich, Switzerland.
}

Correspondence to: Prof Madhulika Kabra, Genetic Unit, Department of Pediatrics, All India Institute of Medical Sciences, New Delhi 110 029, India.madhulikakabra@hotmail.com

Received: June 11, 2011;

Initial review: June 23, 2011;

Accepted: November 12, 2011.
Molecular testing for a specific metabolic disorder remains the gold standard due to its high specificity and sensitivity and possibility of accurate prenatal diagnosis. We report four cases of urea cycle defect where mutational analysis of the involved genes was performed and subsequently, prenatal diagnosis could be offered to one of the family.

Key words: Citrullinemia, Inborn errors of metabolism, Ornithine transcarbomylase deficiency.
U rea cycle disorders (UCD) result from defects in the metabolism of the surplus nitrogen produced by the breakdown of protein and other nitrogen containing molecules. Newborns with a urea cycle disorder often appear normal during the first few days of life but rapidly develop hyperammonemic cerebral edema and related signs of lethargy, anorexia, hyperventilation or hypoventilation, hypothermia, seizures, abnormal posturing, and coma. In milder (or partial) urea cycle enzyme deficiencies, the first recognized clinical episode may be delayed for months, years or decades. Here,ammonia accumulation may be triggered by illness or stress at almost any time of life. These hyperammonemic episodes are marked by loss of appetite, cyclic vomiting, lethargy, and behavioral abnormalities. We herein report four cases of varying severity with various enzyme defects in urea cycle where molecular diagnosis later was helpful in counseling and prenatal diagnosis.

\section{Case Reports}

Case 1: A six-year-old male child presented with developmental delay and seizures since the age of three months. His perinatal history was uneventful. Examination showed presence of microcephaly (Head circumference $49 \mathrm{~cm}<3^{\text {rd }}$ centile by NCHS), slurred speech, clumsiness while walking and writing. He had increased tone and brisk reflexes. His MRI showed cerebral atrophy. He had hyperammonemia (levels between 200-250 $\mu \mathrm{mol} / \mathrm{L}$, normal $<80 \mu \mathrm{mol} / \mathrm{L}$ ) intermittently for which he was on sodium benzoate therapy. Tandem mass spectroscopy showed high citrulline levels. Mutation analysis of the ASS1 gene was carried out by direct DNA sequencing (ABI 3100, 4 capillary sequencer using Big dye terminator version 3.1 chemistry). It revealed a mutation p.Arg265Cys in a homozygous state. Unfortunately, the child developed intractable seizures, chronic encephalopathy which progressed to coma and death at 10 years of age.

Case 2: A four-day-old female born to a nonconsanguinous couple was admitted with neonatal encephalopathy on day three of life. There was history of previous sib death with similar complaints. Investigations of this child showed hyperammonemia ( $>236 \mu \mathrm{mol} / \mathrm{L}$ ) on several occasions without significant acidosis. Her tandem mass spectrometry (TMS) showed very high levels of citrulline $(>5000 \mu \mathrm{mol} / \mathrm{L})$, urine thin layer chromatography also showed increased citrulline. She responded dramatically to ammonia lowering medications (Sodium benzoate) and peritoneal dialysis. Subsequently, child was lost to follow up but her DNA analysis of the ASS1 gene showed the mutation p.Arg157His in a homozygous state with both parents being carriers for the same mutation.

Case 3: Another four day-old female child, a product of 
nonconsanguinous couple presented with refusal to feed, and seizures. She had hyperammonemia (>295 $\mu \mathrm{mol} / \mathrm{l})$. There was no metabolic acidosis. Baby died at the age of 1 week. There was history of previous sib death on day 3 of life with similar complaints. TMS was suggestive of citrullinemia. DNA analysis of the ASS1 gene showed the mutation p.Gly390Arg in a homozygous state and parents were found to be the carrier of the same mutation. In a next pregnancy, the fetus was tested for the same mutation and was found to be only a carrier, so the couple continued with the pregnancy and gave birth to a healthy male child. The child is now one year old and doing well.

Case 4: Three day old male baby was admitted in neonatal ICU with recurrent episodes of seizures and encephalopathy. He was born to a non-consanguineous couple and had an uneventful antenatal and perinatal period. There was history of a male sib death at the age of 5 days with neonatal encephalopathy but no diagnosis could be made. This baby had a negative septic screen and normal CSF but had ammonia levels of 177-236 ìmol/1 on multiple occasions. Blood gas analysis showed no acidosis. The baby received sodium benzoate for hyperammonemia and peritoneal dialysis was also done but encephalopathy did not improve and the baby died on day 5 of life. Urine gas chromatography mass spectroscopy (GCMS) for orotic acid could not be done as he remained anuric since admission to the intensive care unit. However his TMS showed very low citrulline, normal arginine and borderline ornithine. Mutation analysis of the OTC gene using DNA from blood and direct sequencing showed a novel hemizygous mutation in exon 9 of the OTC gene, namely c.988_c.989delAG (fs352X) with the mother being heterozygous for this mutation.

\section{Discussion}

In this cohort of patients, urea cycle defect was suspected on the basis of clinical presentation, family history and basic metabolic workup including arterial blood, gases, blood ammonia, lactate, TMS and GCMS. Diagnosis was confirmed by DNA analysis on the saved blood samples from the proband. Two were diagnosed as classical citrullinemia [1] in the neonatal period (Case 2,3) and third was diagnosed at the age of 6 years (Case 1) as milder late onset form. Molecular diagnosis can be done by direct sequence analysis of the ASS1 gene containing 16 exons. Till date, 87 mutations have been found, distributed throughout exons 3 to 15 , most of them being identified in exons 5,12,13, and 14 and in several intervening sequences, leading to abnormal messenger RNA splicing. The mutation G390R in exon 15 is the single most common mutation in patients with the classical phenotype [2]. Mild, or late-onset citrullinemia type I was found to be associated with a number of specific mutations [2]. Two of our early onset patients had R157H (case 2) G390R (case 3) mutation respectively whereas the patient with milder form had R265C mutation. All of these mutations have been previously reported in the literature. Based on the known mutational profile in one family (Case 3) prenatal diagnosis by direct mutation testing on chorionic villi was done and the fetus was found to be a carrier.

The single most common mutation that has been found in patients with classical citrullinemia is G390R which was observed in our patient (case 3) also. Other two mutations $\mathrm{R} 157 \mathrm{H}$ and $\mathrm{R} 265 \mathrm{C}$ respectively have also been described in patients with severe phenotype [2]. However, in our case R265C mutation was observed in patient with milder phenotype suggesting that the genotype phenotype correlation may not be very strong.

In family 4 diagnosis of ornithine transcarbamylase deficiency was suspected based on the clinical presentation and extensive metabolic workup. A molecular diagnosis was only possible as the DNA was stored from the deceased affected child. It is a novel mutation and was not found in 100 normal controls. However, expression studies were not done. Now, this family can also be provided prenatal diagnosis especially since the mother was also found to be a carrier of this mutation.

Specificity of various screening investigations in diagnosis of IEM may not be very high and enzymatic diagnosis is routinely not available. Moreover, using metabolites as a method of prenatal diagnosis has its own limitations and is not widely available. Hence, molecular studies wherever possible should be carried out. Specific molecular diagnosis is helpful in counseling and prenatal diagnosis for future pregnancies.

Acknowledgment: Prof Dr Olaf Bodamer, Wien.

Contributors: NG collected data, drafted the paper and finalized the manuscript. MK revised the manuscript for important intellectual content. She will act as guarantor of the study. JH conducted the DNA tests, and interpreted them. The final manuscript was approved by all authors.

Funding: ICMR; Competing interests: None stated.

\section{REFERENCES}

1. Brusilow SW, Horwich AL. Urea cycle enzymes. In: Scriver C, Beaudet A, Valle D, Sly W, eds. Metabolic and Molecular Bases of Inherited Disease. New York: McGraw Hill. 2001;1909-63.

2. Engel K, Höhne W, Häberle J. Mutations and polymorphisms in the human argininosuccinate synthetase (ASS1) gene. Hum Mutat. 2009;30:300-7. 